\title{
Influence of Game Evolution and the Phase of Competition on Temporal Game Structure in High-Level Table Tennis Tournaments
}

\author{
by \\ Jorge Vieira de Mello Leite', Fabio Augusto Barbieri², Willian Miyagi², \\ Elvis de Souza Malta², Alessandro Moura Zagatto ${ }^{2}$
}

\begin{abstract}
The aims of this study were: a) to investigate the game temporal structure in high-level table tennis competitions; $b$ ) to verify the influence of game evolution in international competitions from 2009 to 2012 (World Table Tennis Championships and the Olympic Games) on game temporal structure; $c$ ) to compare game temporal structure according to the phase of competition. Comparisons between the three international tournaments demonstrated that rally duration decreased significantly $(p<0.05)$ during the analyzed period $(2009-2012)$, while the rest time increased $(p<0.05)$ from 2009 to 2011, but decreased ( $p<0.05)$ from 2011 to 2012. In the competition phase analysis, it was found that rally duration decreased $(p<0.05)$ in the quarterfinals in relation to the semifinals and finals, while the rest time increased $(p<0.05)$ from the quarterfinals to semifinals and finals. Based on our findings and previous literature, we concluded that the performance level, game evolution and the competition phase influenced the game temporal structure of table tennis, considering longer rest periods adopted by elite athletes in relation to non-elite athletes, the reduction in rally duration and an increase in rest time over the 2009-2012 period and through the competition phases (quarterfinals to finals).
\end{abstract}

Key words: table tennis, game temporal analysis, rally duration, international tournaments.

\section{Introduction}

Game temporal characteristics have been widely studied in racket sports such as tennis, badminton, squash and table tennis, which can be good references for specific training prescription (Cabello Manrique and González-Badillo, 2003; Fernandez-Fernandez et al., 2007; Kovacs, 2006; Lees, 2003; Zagatto et al., 2010). Although some studies have investigated temporal variables in high-level table tennis matches (Drianovski and Otcheva, 2002; Katsikadelis et al., 2007; Katsikadelis et al., 2010; Zagatto et al., 2016), these studies analyzed tournaments conducted until 2008, i.e. the time when the International Table
Tennis Federation (ITTF) banned the speed glue, a tool that was used to increase the elasticity of the rubber and add speed and spin to the ball. Only one study (Zagatto et al., 2010) analyzed temporal variables in official matches considering the prohibition of speed glue. The authors found total match duration of $16 \mathrm{~min}$, rally duration of $3.4 \mathrm{~s}$, rest time between rallies of $8.1 \mathrm{~s}$ and an effort and rest ratio of the match of 0.4 (Zagatto et al., 2010). Recently Zagatto et al. (2016) also reported similar values for rally duration (3.5 s), rest time between rallies $(8.2 \mathrm{~s})$ and an effort and rest ratio of the match (0.44), but measured during simulated

\footnotetext{
1 - Health and Development Postgraduate Program, Federal University of Mato Grosso do Sul (UFMS), Campo Grande, Brazil.

2 - Department of Physical Education, São Paulo State University (Unesp), School of Sciences, Bauru-SP, Brazil.
} 
table tennis games. Therefore, a game temporal structure analysis in high-level table tennis competitions could provide important information for training prescription, especially if the analysis considers the game evolution over the years and different phases of the competition.

However, the improvement of the racket and rubber technologies over time (Zoltan and Reinhold, 2007), change of the ball size from 38 to $40 \mathrm{~mm}$ (a new $40 \mathrm{~mm}$ ball was introduced after the 2000 Summer Olympics) (Iimoto et al., 2002; Ishigaki, 2002; Takeuchi et al., 2002; Tang et al., 2002) and the ban on speed glue (Nicklas et al., 2007; Tsuji et al., 2007) might have influenced the game temporal structure. In addition, rule changes implemented by the ITTF to the score system (from 21 to 11 points) and new serving requirements (the adoption of the non-hidden service, in which players cannot hide the ball from the opponent during the service) have induced the players to evolve their techniques and tactics over the years (Li et al., 2005; Zhang et al., 2013) which may also have influenced the game temporal structure. Regarding the phase of competition, it is known that the final round of most competitions involves the best table tennis players, which could change the game temporal structure compared to other phases of the competition (i.e., quarterfinals and semifinals). Finally, the balanced disputes of the final phase may require more attention to tactical and psychological aspects, which also may have impact on temporal variables.

Therefore, in order to obtain more accurate information to support the training prescription for elite table tennis players, the aims of this study were: a) to investigate the game temporal structure in high-level table tennis competitions; b) to verify the influence of game evolution in international competitions from 2009 to 2012 (the 2009 World Table Tennis Championships, the 2011 World Table Tennis Championships and the 2012 Olympic Games) on game temporal structure; c) to compare the game temporal structure according to the phase of competition (quarterfinals, semifinals and finals). We hypothesized that the temporal structure in elite level table tennis competitions would be different from the Brazilian tournaments of a national and regional level (Zagatto et al., 2010) performed by tennis players of various sports levels (Fernandez et al., 2006), and that the game evolution over the years and the phase of competition would modify game temporal structure in high-level table tennis tournaments.

\section{Material and Methods}

\section{Sample matches}

The analysis of game temporal structure was performed using videos from 21 official matches played by 16 elite male table tennis players (age $29.5 \pm 3.8$ years, $68.7 \%$ right-handed and $31.3 \%$ left-handed, $75 \%$ Asian and $25 \%$ European). The majority of the athletes (75\%) were among the top 10 in the world ranking while $37.5 \%$ had achieved the first place of the world ranking at least once in their career. The 21 matches selected corresponded to the quarterfinals (12 matches, 62 sets), semifinals (6 matches, 33 sets) and finals ( 3 matches, 15 sets) of the 2009 World Table Tennis Championships (7 matches, 38 sets), the 2011 World Table Tennis Championships (7 matches, 37 sets) and the 2012 Olympic Games (7 matches, 35 sets). Among the selected matches, nine were disputed between Chinese table tennis players, which represented $100 \%$ of the finals, $50 \%$ of the semifinals and $25 \%$ of the quarterfinals. The procedures had been previously approved by the Ethics Committee on Human Research of the Federal University of Mato Grosso do Sul - Brazil (CAAE: 10499512.0.0000.0021/2012) and were conducted in accordance with the Declaration of Helsinki.

\section{Game temporal structure analysis}

The analysis of the game temporal structure was performed using Windows Media Player software (version 12.0, Microsoft, Redmond, Washington, USA) from video recordings. From the videos, the duration of each rally (an effort period) and each rest time between rallies (a recovery period) were measured individually in all matches using clocks with chronometers (Marathon 50 Lap Memory Stopwatch T5G811, Timex, USA). The duration of each rally was determined using the time recorded from the last moment at which the ball was immobile on the palm of the free hand before being intentionally projected to the service execution until the end of the rally, which was always decided as a 'let' service rally (the ball touched the net before falling to the receiver side, which required the repetition of the point) or as a 
point won by one of the athletes (International Table Tennis Federation, 2013; Zagatto et al., 2010). The duration of rest time was measured from the end of the rally until the beginning of the next serve. The procedures used to measure the duration of the rally and rest time between rallies are highly reproducible and demonstrate high intraclass correlations (ICC $=0.78, p<0.001$ for the rally and ICC $=0.95, p<0.001$ for rest time) (Zagatto et al., 2010).

From the measurement of rally duration and rest time between rallies, it was possible to determine the effort and rest ratio of the match and, within the sets, total match duration, total set duration, real playing time of the match and each set as well as effective playing time of the match and each set (Zagatto et al., 2010). The real playing time of the match and each set was obtained by summing the duration of all rallies of the match and set, respectively. The effective playing time of the match was determined by the ratio of the real playing time in the match and total match duration. The effective playing time (EPT) of the set was calculated using a similar formula.

\section{EPT $=($ real playing time/ total match duration $) \times 100$}

The number of shots per rally and the number of rallies per match and per set were also determined. The 'let' service rallies were disregarded in the rally duration calculations, while the technical times requested by coaches during matches and the intervals between sets were not included in the determination of rest time between rallies. All these temporal variables were determined individually for each selected match.

\section{Data analysis}

A descriptive statistical analysis was used for determination of the temporal variables in the selected matches. The results were presented as median, range and quartiles ( $1^{\text {st }}$ and $3^{\text {rd }}$ ). The Kolmogorov-Smirnov and Shapiro-Wilk tests were used to evaluate the data normality of variables with more than 50 and less than 50 samples respectively. The nonparametric KruskalWallis test with a post hoc Dunn test were used to compare the temporal variables between three events (the 2009 World Table Tennis Championships, the 2011 World Table Tennis Championships and the 2012 Olympic Games) and between three competition phases (quarterfinals, semifinals and finals) for variables that were not normally distributed, while the oneway ANOVA with a post hoc Tukey test was used to perform the same comparisons in variables that presented normal distribution. All analyzes were performed using IBM SPSS statistical package for Windows (Version 20.0, Armonk, New York, United States), adopting a significance level of $5 \%$ $(p<0.05)$.

\section{Results}

The game temporal analysis of the 21 matches is presented in Table 1. It showed that rally duration was $3.3 \mathrm{~s}$, while the rest time between rallies was $18.6 \mathrm{~s}$, resulting in 0.18 for the effort and rest ratio in a match. The total duration of matches was $\sim 45 \mathrm{~min}$ and around 88 points were played in each match.

Regarding the events comparison, statistical analyses demonstrated that rally duration and rest time between rally values were significantly different between the three international events, that rally duration decreased from 2009 to 2011 and from 2011 to $2012(\mathrm{H}(2.0)=$ 63.26; $p<0.001)$, while the rest time between rallies increased from 2009 to 2011, but decreased from 2011 to $2012(\mathrm{H}(2.0)=66.16 ; p=0.0001)$. The effort and rest ratio in a match $(p=0.20)$, total match duration $(p=0.78)$ and effective playing time $(p=0.10)$ in a match were not different between competitions, but the effort and rest ratio in a set $(\mathrm{H}(2.0)=24.38 ; p<0.001)$ and effective playing time in a set $(\mathrm{H}(2.0)=18.00 ; p<0.001)$ were statistically higher in the 2009 World Championships in relation to the 2011 World Championships and the 2012 Olympic Games (Table 2).

Regarding the game temporal structure according to the phase of competition, a significant reduction was found in rally duration from the quarterfinals to the semifinals and finals $(\mathrm{H}(2.0)=31.71 ; p<0.001)$, while the rest time between rallies increased from the quarterfinals to the semifinals and finals $(\mathrm{H}(2.0)=91.33 ; p<0.001)$. These results were followed by a significant decrease in the effort and rest ratio in a set $(\mathrm{H}(2.0)$ $=28.33 ; p<0.001)$ and effective playing time in a set $(\mathrm{H}(2.0)=25.38 ; p<0.001)$ from the quarterfinals to the semifinals and finals (Table 3 ). 


\section{Table 1}

Medians ( $1^{\text {st }}$ quartile-3 ${ }^{\text {rd }}$ quartile) and ranges of the game temporal structure variables obtained in all table tennis matches $(n=21)$.

\begin{tabular}{|c|c|c|}
\hline & Median (1 ${ }^{\text {st }}$ quartile $-3^{\text {rd }}$ quartile) & Range \\
\hline $\mathrm{RD}(\mathrm{s})$ & $3.3(2.7-4.4)$ & $1.1-31.2$ \\
\hline $\mathrm{RT}(\mathrm{s})$ & $18.6(14.5-23.4)$ & $1.8-93.2$ \\
\hline NSR (shots·rally ${ }^{-1}$ ) & $4.0(3.0-6.0)$ & $1.0-38.0$ \\
\hline $\mathrm{NRM}\left(\right.$ rallies $\cdot$ match $\left.^{-1}\right)$ & $88.0(82.0-113.0)$ & $60.0-134.0$ \\
\hline NRS (rallies·set ${ }^{-1}$ ) & $18.0(16.0-20.0)$ & $12.0-34.0$ \\
\hline E:RMATCH & $0.18(0.15-0.21)$ & $0.14-0.28$ \\
\hline E:RsET & $0.19(0.15-0.23)$ & $0.10-0.41$ \\
\hline TMD (s) & $2708.0(2232.6-3388.3)$ & $1182.3-4208.9$ \\
\hline TSD (s) & $434.6(355.0-544.9)$ & $195.6-888.5$ \\
\hline RPTMATCH (s) & $366.3(326.2-441.7)$ & $240.0-603.0$ \\
\hline RPTSET(s) & $73.0(63.57-84.4)$ & $40.7-121.1$ \\
\hline ЕРТМАТсH $(\%)$ & $14.7(11.7-15.9)$ & $10.6-24.2$ \\
\hline EPTSET $(\%)$ & $16.5(13.9-19.9)$ & $7.6-30.0$ \\
\hline
\end{tabular}

Abbreviations: $R D=$ rally duration, $R T=$ rest time between rallies, $N S R=$ number of shots per rally, NRM = number of rallies per match, NRS = number of rallies per set, $E: R_{M A T C H}=$ effort and rest ratio in a match, E:RSET = effort and rest ratio in a set, TMD = total match duration, TSD = total set duration, $R P T_{\text {MATCH }}$ real playing time in a match, $R P T_{S E T}=$ real playing time in a set, EPT $T_{M A T C H}=$ effective playing time in a match, EPTSET = effective playing time in a set.

Table 2

Medians (1 ${ }^{\text {st }}$ quartile-3 ${ }^{\text {rd }}$ quartile) of temporal variables of game structure variables according to competition: the 2009 World Championships, the 2011 World

Championships and the 2012 Olympic Games.

\begin{tabular}{|c|c|c|c|}
\hline & $\begin{array}{l}2009 \text { World Championships } \\
\qquad(n=7)\end{array}$ & $\begin{array}{l}2011 \text { World Championships } \\
\qquad(n=7)\end{array}$ & $\begin{array}{c}2012 \text { Olympic Games } \\
(n=7)\end{array}$ \\
\hline $\mathrm{RD}(\mathrm{s})$ & $3.6(2.9-4.9)$ & $3.3(2.7-4.3)^{*}$ & $3.1(2.5-4.0)^{*} q$ \\
\hline $\mathrm{RT}(\mathrm{s})$ & $17.0(13.4-21.2)$ & $20.2(16.1-25.1)^{*}$ & $18.9(14.7-23.6)^{*} q$ \\
\hline NSR (shots $\cdot$ rally $^{-1}$ ) & $5.0(3.0-7.0)$ & $4.0(3.0-6.0)^{*}$ & $4.0(3.0-5.3)^{*}$ \\
\hline $\mathrm{NRM}\left(\right.$ rallies $\left.\cdot \mathrm{match}^{-1}\right)$ & $102.0(85.0-117.5)$ & $84.0(81.0-114.0)$ & $88.0(73.5-104.0)$ \\
\hline NRS (rallies:set ${ }^{-1}$ ) & $19.0(17.0-20.0)$ & $18.0(16.0-20.0)$ & $17.0(15.0-20.0)$ \\
\hline E:RмAтсH & $0.21(0.19-0.23)$ & $0.16(0.15-0.19)$ & $0.15(0.15-0.22)$ \\
\hline E:RsET & $0.22(0.19-0.26)$ & $0.16(0.14-0.19)^{*}$ & $0.17(0.14-0.20)^{*}$ \\
\hline TMD (s) & $2724.0(2280.0-2994.0)$ & $2646.0(2388.0-3522.0)$ & $2706.00(1872.0-3078.0)$ \\
\hline $\operatorname{TSD}(\mathrm{s})$ & $450.0(378.0-558.0)$ & $432.0(384.0-534.0)$ & $438.0(300.0-552.0)$ \\
\hline RPTМАTCH (s) & $390.0(366.0-582.0)$ & $348.0(324.0-432.0)$ & $324.0(288.0-360.0)$ \\
\hline RPTSET(s) & $84.0(72.0-96.0)$ & $72.0(60.0-78.0)^{*}$ & $66.0(60.0-78.0)^{*}$ \\
\hline ЕРТМАТсн $(\%)$ & $15.7(15.0-17.4)$ & $12.7(11.6-14.3)$ & $12.0(11.6-15.3)$ \\
\hline EPTset $(\%)$ & $19.2(16.8-21.8)$ & $15.1(13.8-17.1)^{*}$ & $14.8(13.1-18.4)^{*}$ \\
\hline
\end{tabular}

Abbreviations: $\mathrm{RD}=$ rally duration, $\mathrm{RT}=$ rest time between rallies, NSR = number of shots per rally, NRM = number of rallies per match, NRS = number of rallies per set, E:RMATCH = effort and rest ratio in a match, E:RSET = effort and rest ratio in a set, TMD = total match duration, $T S D=$ total set duration, $R P T_{M A T C H}=$ real playing time in a match, $R P T_{S E T}=$ real playing time in a set, EPTMATCH $=$ effective playing time in a match, EPTSET = effective playing time in a set.

* Different from 2009; q Different from 2011 


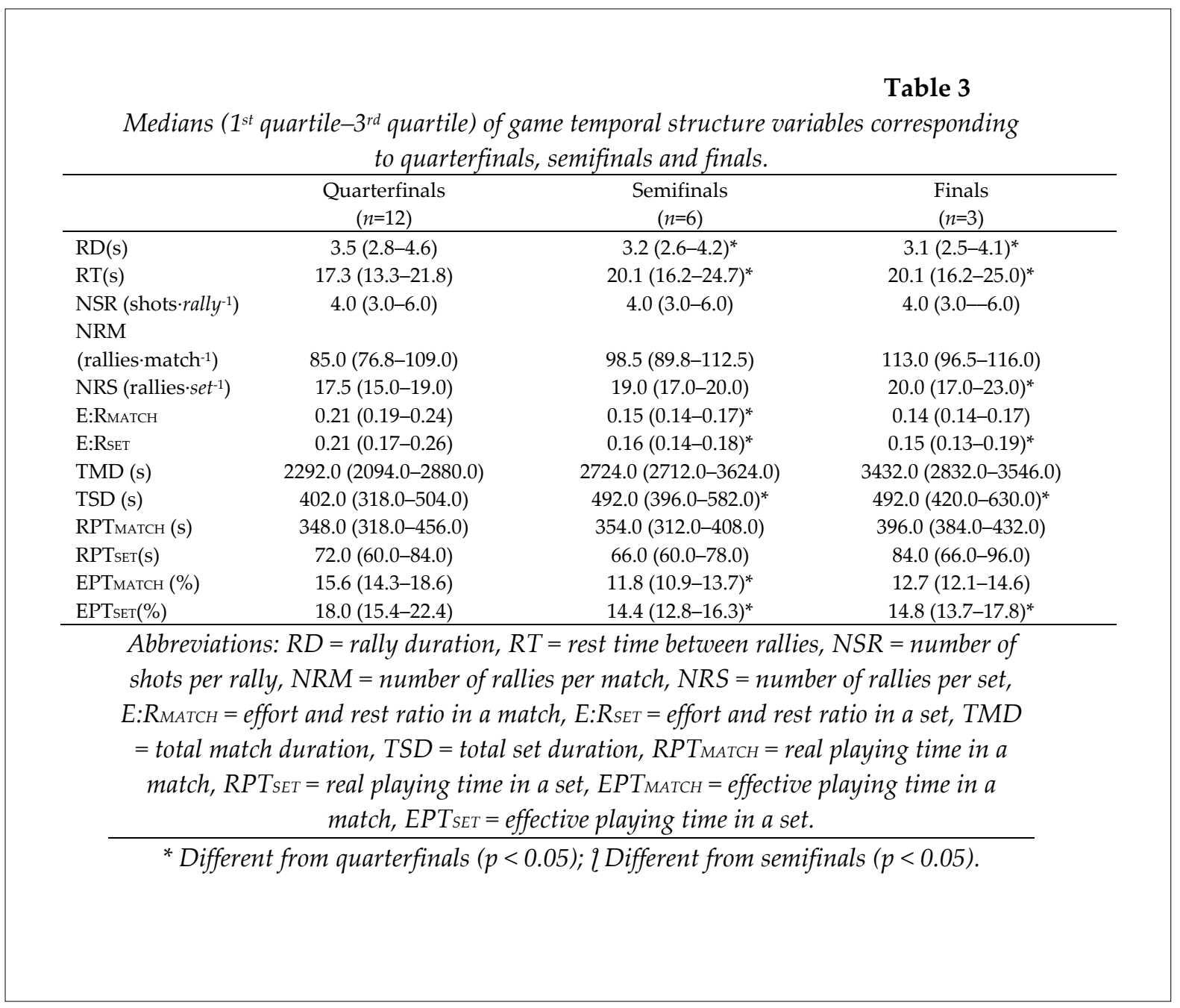

\section{Discussion}

According to the authors' knowledge, this is the first study to investigate the temporal structure of official matches disputed between elite table tennis players in international events organized under the current rules of the ITTF. It is important to emphasize that the selected matches were played between elite table tennis players, considering that $75 \%$ of them belonged to the top 10 world ranking and $37.5 \%$ had achieved the $1^{\text {st }}$ place of the world ranking at least once. The main findings demonstrated that the duration of rallies and rests of the analyzed matches were $3.3 \mathrm{~s}$ and 18.6 s respectively, while the effort and rest ratio in a match was 0.18. Comparisons between the three international tournaments demonstrated that rally duration decreased significantly during the analyzed period (2009-2012), while the rest time increased from 2009 to 2011, but decreased from 2011 to 2012. In the temporal analysis of competition phases, it was found that rally duration decreased from the quarterfinals in relation to the semifinals and finals, while rest time increased from the quarterfinals to the semifinals and finals. In the following paragraphs, we will discuss some interpretations in terms of temporal structure of official matches disputed between elite table tennis players and explanations of the temporal structure differences found between the three international tournaments and competition phases.

\section{Game temporal structure in elite table tennis matches}

Contrary to our first hypothesis, our findings were similar to those of a previous study (Zagatto et al., 2010), which presented $3.2 \mathrm{~s}$ and $3.6 \mathrm{~s}$ for the rally duration in official matches played under the current rules of the ITTF 
between Brazilian table tennis players of a national and regional level, respectively. This finding suggests that the rally duration in table tennis competitions is not influenced by the athlete's level. However, in accordance with our first hypothesis, we found that elite table tennis players adopted longer intervals of rest (18.6 s) compared to athletes at national $(9.3 \mathrm{~s})$ and regional (7.0 s) level (Zagatto et al., 2010), which resulted in slightly lower values of the effort and rest ratio in a match (0.18) and effective playing time in a match (14.7\%) at international tournaments in relation to national competitions (0.3 and $21.4 \%$ ) and considerably lower values than at regional tournaments (0.5 and 60.7\%) (Zagatto et al., 2010). These results suggest a direct relationship between the level of performance and temporal variables, such that when the athlete's level is higher, the effort and rest ratio and effective playing time in a match will be lower due to longer rest periods employed between efforts. Previous studies showed that table tennis players often use several strategies to cope with stress, regulate arousal and control anxiety (Iizuka et al., 2005; Leite et al., 2012; Lima et al., 2004). Thus, it is plausible to suggest that these temporal differences between athletes with different performance levels are due to longer rest periods adopted by elite table tennis players to adjust psychological aspects.

When comparing our results with the findings obtained in elite athletes of other racket sports, it was noted that the rally duration in table tennis (3.3 s) was shorter than in tennis (7.2 to 10.2 s) (Christmass et al., 1998; Fernandez-Fernandez et al., 2008; Mendez-Villanueva et al., 2007), squash (13.6 and 18.6 s) (Girard et al., 2007; Montpetit, 1990) and badminton (6.4 s) (Cabello Manrique and González Badillo, 2003). On the other hand, the rest times measured in squash ( $>$ 9 s) (Girard et al., 2007; Montpetit, 1990), badminton ( $\sim 13 \mathrm{~s})$ (Cabello Manrique and González Badillo, 2003) and tennis (15.5 to $16.8 \mathrm{~s}$ ) (Christmass et al., 1998; Fernandez-Fernandez et al., 2008; Mendez-Villanueva et al., 2007) were shorter than in table tennis ( 19 s). These differences resulted in a considerably lower effort and rest ratio of a match in table tennis (0.18) compared to tennis (0.47 to 0.58) (Christmass et al., 1998; Fernandez-Fernandez et al., 2008; Mendez-Villanueva et al., 2007), badminton (0.49)
(Cabello Manrique and González Badillo, 2003) and squash (2.4) (Girard et al., 2007). Thus, although there are similarities between racket sports, sport specific temporal variables should be considered in training for table tennis. The training prescription in table tennis should not consider the results of temporal variables obtained in other racket sports such as tennis, squash or badminton.

Effects of game evolution and competition phases on game temporal structure of elite table tennis matches

Our findings confirmed our second hypothesis as the game evolution over the years (2009-2012) and the competition phase interfered in game temporal structure. Regarding the significant decrease in rally duration from the 2009 World Championships (3.6 s) to the 2011 World Championships (3.3 s) and the 2012 Olympic Games (3.1 s), previous authors indicated that the use of a new backhand technique to attack short balls (close to the net) motivated elite table tennis players to receive the opponent's service in an offensive way more often in recent years, which may have contributed to the faster finalization of rallies in the 2011 and 2012 tournaments (Huang and Zhang, 2013). Concerning the rest time differences between the events, we were not able to indicate the main reasons for the higher rest time found in 2011 compared to the other events. However, we believe that the athletes who participated in the 2011 World Championships adopted longer rest time between rallies for several reasons, including attempts to adjust psychological and tactical factors. In addition, comparing our findings $(3.3 \mathrm{~s}$ for the rally and $18.6 \mathrm{~s}$ for rest) and the results obtained for matches of elite table tennis players at the 1998 University World Championships (3.6 $\mathrm{s}$ for the rally and $9.4 \mathrm{~s}$ for rest) (Drianovski and Otcheva, 2002), we noted that the rally duration did not change significantly between 1998 and the period of the analyzed events (2009-2012). However, the rest time increased by approximately $9 \mathrm{~s}$, demonstrating that the temporal structure of elite table tennis has changed over the years due to the game evolution. This change was probably influenced by alterations implemented by the ITTF in the score system in 2001 (from 21 to 11 points in each set) and the adoption of the non-hidden service in 
2002 (players were prohibited from hiding the ball during the service using their body or a free arm), because these two modifications in the rules compelled players to adjust their techniques and tactics (Li et al., 2005; Zhang et al., 2013).

In the 11-point score system, the outcome of the game became more unexpected, which increased the mental pressure on elite table tennis players, furthermore, as a result of the introduction of the non-hidden service, the receiving players began to see clearly the full trajectory of the opponent's serve, which increased the possibility of performing immediate attacks in reception (Li et al., 2005). Therefore, it is possible that in current elite tournaments the table tennis players adopt longer pauses between rallies due to the higher pressure and greater importance attributed to each point in the 11-point system, besides the need to use more time for continual reflection about tactical aspects involving the nonhidden service (spin, placement, speed, height, unpredictability) to prevent offensive receptions.

Our results confirmed that the competition phase interfered in the game temporal structure, which demonstrated a significant decrease in rally duration from the quarterfinals ( $3.5 \mathrm{~s})$ to the semifinals $(3.2 \mathrm{~s})$ and finals (3.1 s), while the rest time increased from the quarterfinals $(17.3 \mathrm{~s})$ to the semifinals $(20.1 \mathrm{~s})$ and finals (20.1 s). It is possible that this reduction in rally duration through the phases was influenced by the higher number of matches between Chinese athletes in the semifinals (50\% of six matches) and finals (100\% of three matches) compared to the quarterfinals $(25 \%$ of 12 matches). This is likely because Chinese table tennis players have better technique effectiveness in the strokes used at the beginning of a rally (serve, reception, $3^{\text {rd }}$ and $4^{\text {th }}$ stroke) compared to elite table tennis players from other countries and regions, which may have resulted in points being finalized more quickly in the semifinals and finals (Zhang et al., 2013). To explain the increase in the rest time, we noted a tendency for more rallies to be disputed in the final matches (113 points) compared to the semifinals ( 98 points) and quarterfinals (85 points), suggesting greater balance and competitiveness in the final two phases of competitions. Thus, it is possible that the table tennis players used longer rest periods between rallies due to balanced disputes, which require more attention to tactical and psychological issues.

However, the present study has some limitations. First of all, effectiveness of the technique performed during the rally was not investigated; furthermore, we did not evaluate the effect of the playing style on temporal variables of game structure. The aforementioned aspects shall be considered in future studies focused on notational analysis in table tennis

\section{Conclusion}

Due to the differences found in several temporal variables in matches played between athletes of different levels, between three elite tournaments realized at different dates (2009, 2011 and 2012) and between competition phases, we concluded that i) game temporal structure was influenced by the athlete's level; ii) the game evolution and the competition phase changed game temporal structure, reducing rally duration and increasing rest time periods. Therefore, the findings of this study should be used to support training prescription for elite table tennis players, in a way that the effort and rest ratio, rally duration and rest time are used as very applicable tools to develop specific technical and physical exercises. Coaches can use stopwatches and metronomes to control training, especially in multi-ball sessions which require a coach or a machine throwing a pre-determined number of balls per minute to the athletes.

\section{Acknowledgements}

Our acknowledgements to Professor Fernandes for the explanations about the data analysis.

Laboratory where the research was conducted: Laboratory of Exercise Physiology and Human Performance (LAFIDE) - Department of Physical Education, Sao Paulo State University (Unesp), School of Sciences, Av. Eng. Luiz Edmundo Carrijo Coube, 14-01, Vargem Limpa, CEP 17033-360 Bauru-SP, Brazil. 


\section{References}

Cabello Manrique D, González-Badillo JJ. Analysis of the characteristics of competitive badminton. $\mathrm{Br} J$ Sports Med, 2003; 37: 62-66

Christmass MA, Richmond SE, Cable NT, Arthur PG, Hartmann PE. Exercise intensity and metabolic response in singles tennis. J Sports Sci, 1998; 16: 739-747

Drianovski Y, Otcheva G. Survey of the game styles of some of the best Asian players at the $12^{\text {th }}$ World University Table Tennis Championship (Sofia, 1998). Int J Table Tennis Sci, 2002; 4: 1-7

Fernandez J, Mendez-Villanueva A, Pluim BM. Intensity of tennis match play. Br J Sports Med, 2006; 40: 387391

Fernandez-Fernandez J, Mendez-Villanueva A, Fernandez-Garcia B, Terrados N. Match activity and physiological responses during a junior female singles tennis tournament. Br J Sports Med, 2007; 41: $711-716$

Fernandez-Fernandez J, Sanz-Rivas D, Fernandez-Garcia B, Mendez-Villanueva A. Match activity and physiological load during a clay-court tennis tournament in elite female players. J Sports Sci, 2008; 26: 1589-1595

Girard O, Chevalier R, Habrard M, Sciberras P, Hot P, Millet GP. J Strength Cond Res, 2007; 21: 909-914

Huang W, Zhang H. The effect analysis of Zhang Jike's return serve with backhand side-twisted at the 2/3 court right - 2012 London Olympic table tennis singles. Int J Table Tennis Sci, 2013; 8: 72-76

Iimoto Y, Yoshida K, Yuza N. Rebound characteristics of the new table tennis Ball; Differences between the $40 \mathrm{~mm}(2.7 \mathrm{~g})$ and $38 \mathrm{~mm}(2.5 \mathrm{~g})$ balls. Int J Table Tennis Sci, 2002; 5: 233-243

lizuka CA, Dantas LEPBT, Machado AA, Marinovic W. Anxiety control in table tennis players and their relation with sportive performance. Rev Mackenzie Ed Fís Esporte, 2005; 4: 127-135

International Table Tennis Federation (ITTF). The Laws of Table Tennis, ITTF Regulation Handbook, 2013. Available at: http://www.ittf.com/ittf_handbook; accessed on: 01.04.2015

Ishigaki H. Distribution of contact points on the racket when hitting 40mm balls. Int J Table Tennis Sci, 2002; 5: $244-250$

Katsikadelis M, Pilianidis T, Vasilogambrou A. Real play time in table tennis matches in the XXVIII Olympic games «Athens 2004». Int J Table Tennis Sci, 2007; 15: 1-5

Katsikadelis M, Pilianidis T, Misichroni A. Comparison of Rally Time in XXIX Beijing (2008) and XXVIII Athens (2004) Olympic Table Tennis Tournaments. Int J Table Tennis Sci, 2010; 6: 55-59

Kovacs MS. Applied physiology of tennis performance. Br J Sports Med, 2006; 40: 381-385

Lees A. Science and the major racket sports: a review. J Sports Sci, 2003; 21: 707-732

Leite JVM, Madrid B, Bezerra RFA. Arousal physiological and cognitive regulation in table tennis, Rev Motricidade, 2012; 8: 1089-1096

Li JL, Zhao X, Zhang CH. Changes and development: Influence of new rules on table tennis techniques. Int J Table Tennis Sci, 2005; 11: 67-72

Lima FV, Samulski DM, Vilani LHP. Non-systematic coping strategies in table tennis critical game situations. Rev Bras Ed Fís Esporte, 2004; 18: 363-375

Mendez-Villanueva A, Fernandez-Fernandez J, Bishop D, Fernandez-Garcia B, Terrados N. Activity patterns, blood lactate concentrations and ratings of perceived exertion during a professional singles tennis tournament. Br J Sports Med, 2007; 41: 296-300 
Montpetit RR. Applied physiology of squash. Sports Med, 1990; 10: 31-41

Nicklas G, Kraus T, Tiefenbacher K. Facts about speed glue. Int J Table Tennis Sci, 2007; 23: 1-14

Takeuchi T, Kobayashi Y, Hiruta S, Yuza N. The effect of the $40 \mathrm{~mm}$ diameter ball on table tennis rallies by elite players. Int J Table Tennis Sci, 2002; 5: 267-277

Tang HP, Mizoguchi M, Toyoshima S. Speed and spin characteristics of the $40 \mathrm{~mm}$ table tennis ball. Int J Table Tennis Sci, 2002; 5: 278-284

Tsuji Y, Ushiyama Y, Kimura K. Influence of glue on ball speed. Int J Table Tennis Sci, 2007; 23: 1-11

Zagatto AM, Morel EA, Gobatto CA. Physiological responses and characteristics of table tennis matches determined in official tournaments. J Strength Cond Res, 2010; 24: 942-9

Zagatto AM, Papoti M, Leite JVM, Beneke R. Energetics of table tennis and table tennis specific exercise testing. Int J Sports Physiol Perform, 2016: In press.

Zhang H, Liu W, Hu JJ, Liu RZ. Evaluation of elite table tennis players' technique effectiveness. J Sports Sci, 2013; 32: 70-77

Zoltan M, Reinhold LW. Characterization of the friction behavior of table tennis rubbers. Int J Table Tennis Sci, 2007; 20: 1-8

\section{Corresponding author:}

\section{Alessandro Moura Zagatto}

São Paulo State University (Unesp), Schoof of Sciences, Department of Physical Education - Bauru, SP, Brazil Av. Eng. Luiz Edmundo Carrijo Coube, 14-01 - Vargem Limpa Cep: 17.033-360.

Phone: +55 (11) 966040713.

E-mail: azagatto@yahoo.com.br; 